\title{
On linear versions of some addition theorems
}

\author{
Shalom Eliahou and Cédric Lecouvey \\ LMPA Joseph Liouville, FR CNRS 2956 \\ Université du Littoral Côte d'Opale \\ 50 rue F. Buisson, B.P. 699 \\ F-62228 Calais cedex, France
}

\begin{abstract}
Let $K \subset L$ be a field extension. Given $K$-subspaces $A, B$ of $L$, we study the subspace $\langle A B\rangle$ spanned by the product set $A B=\{a b \mid$ $a \in A, b \in B\}$. We obtain some lower bounds on $\operatorname{dim}_{K}\langle A B\rangle$ and $\operatorname{dim}_{K}\left\langle B^{n}\right\rangle$ in terms of $\operatorname{dim}_{K} A, \operatorname{dim}_{K} B$ and $n$. This is achieved by establishing linear versions of constructions and results in additive number theory mainly due to Kemperman and Olson.
\end{abstract}

\section{Introduction}

Let $G$ be a group, written multiplicatively. Given subsets $A, B \subset G$, we denote by

$$
A B=\{a b \mid a \in A, b \in B\}
$$

the product set of $A, B$. For $A, B$ finite, several results in additive number theory give estimates on the cardinality of $A B$ as a function of $|A|,|B|$. Instances of such results are the Cauchy-Davenport theorem for cyclic groups of prime order, a theorem of Kneser for abelian groups, and theorems of Kemperman and of Olson for possibly nonabelian groups.

In this paper, we consider the following analogous question. Given a field extension $K \subset L$, and finite-dimensional $K$-subspaces $A, B$ of $L$, define

$$
\langle A B\rangle=\text { the } K \text {-subspace spanned by the product set } A B \text { in } L \text {. }
$$


What can be said, then, about the dimension of the subspace $\langle A B\rangle$ ? The main object of this paper is to establish linear analogues, in this new setting, of several results in additive number theory. In particular, we shall obtain nontrivial lower bounds on $\operatorname{dim}\langle A B\rangle$ in terms of $\operatorname{dim} A, \operatorname{dim} B$.

This question has barely been addressed in the literature. What seems to be the so far unique result of this type is due to Hou, Leung and Xiang [1] and is given below. We first recall Kneser's theorem from additive number theory [3, 4].

Theorem 1.1 (Kneser) Let $G$ be an abelian group and let $A, B \subset G$ be finite nonempty subsets. Then

$$
|A B| \geq|A H|+|B H|-|H|,
$$

where $H=\{g \in G \mid g A B=A B\}$ is the stabilizer of $A B$.

The following linear version has been obtained in [1], and has motivated us to further explore the links between additive number theory, linear algebra and field extensions.

Theorem 1.2 (Hou, Leung and Xiang) Let $K \subset L$ be a commutative field extension, and let $A, B \subset L$ be nonzero finite-dimensional $K$-subspaces of $L$. Suppose that every algebraic element in $L$ is separable over $K$. Let $H$ be the subfield of $L$ which stabilizes $\langle A B\rangle$. Then

$$
\operatorname{dim}_{K}\langle A B\rangle \geq \operatorname{dim}_{K} A+\operatorname{dim}_{K} B-\operatorname{dim}_{K} H .
$$

The paper is organized as follows. Several classical results and constructions in additive number theory are recalled in Section 2. In Section 3, we give a new variant of a theorem of Olson. The switch to the field extension setting is performed from Section 4 on, where we give linear analogues of the additive results stated in the preceding sections.

\section{Classical addition theorems}

\subsection{A basic result}

One of the simplest results on the product of two sets in a finite group is the following. Given a subset $X$ of a group $G$, we denote $X^{-1}=\left\{x^{-1} \mid x \in X\right\}$. 
Proposition 2.1 Let $G$ be a finite group. Let $A, B$ be nonempty subsets of G. If $|A|+|B|>|G|$, then $A B=G$.

The proof, informally, goes as follows. For $x \in G$, we have $A^{-1} x \cap B \neq \emptyset$, since $\left|A^{-1} x\right|+|B|=|A|+|B|>|G|$. It follows that $x \in A B$.

Linearizing this result requires a little more work. This is done in Section 6, with various proofs.

\subsection{Theorems of Kemperman and Olson}

Here are a few classical results in additive number theory, to be linearized in subsequent sections. We refer the reader to [5] for proofs.

Theorem 2.2 (Kemperman) Let $A, B$ be two finite subsets of a group $G$. Assume there exists an element $c \in A B$ appearing exactly once as a product $c=a b$ with $a \in A, b \in B$. Then

$$
|A B| \geq|A|+|B|-1 \text {. }
$$

The next result is a nonabelian analogue of Kneser's Theorem 1.1.

Theorem 2.3 (Olson) Let $A, B$ be two finite subsets of a group $G$. There exists a nonempty subset $S$ of $A B$ and a finite subgroup $H$ of $G$ such that

$$
|A B| \geq|S| \geq|A|+|B|-|H|
$$

and either $H S=S$ or $S H=S$.

Here is an immediate corollary.

Theorem 2.4 (Kemperman) Let $G$ be a torsion-free group. Let $A, B$ be nonempty finite subsets of $G$. Then

$$
|A B| \geq|A|+|B|-1 \text {. }
$$

Olson [5] also derived, as a consequence of Theorem 2.2 and 2.3, the following two interesting results. Linear versions are given in Section 9.

Theorem 2.5 (Olson) Let $A, B$ be finite subsets of a group $G$ with $1 \in B$. Then

$$
A B^{2}=A B \text { or }|A B| \geq|A|+\frac{1}{2}|B| .
$$

Theorem 2.6 (Olson) Let $B$ be a finite subset of a group $G$. Then

$$
\left|B^{n}\right|=\left|B^{n+1}\right| \quad \text { or }\left|B^{n}\right| \geq\left|B^{n-1}\right|+\frac{1}{2}|B| .
$$




\subsection{A main tool: the Kemperman transform}

The above results are obtained by cleverly iterating Kemperman transforms, which we now recall.

Let $G$ be a (possibly nonabelian) multiplicative group and $(A, B)$ a pair of nonempty finite subsets of $G$. Let $x$ be any element in $G$. The Kemperman transformed pairs $\left(A^{\prime}, B^{\prime}\right)$ and $\left(A^{\prime \prime}, B^{\prime \prime}\right)$ with respect to $x$ are defined by

$$
\left(A^{\prime}=A \cup A x, B^{\prime}=B \cap x^{-1} B\right)
$$

and

$$
\left(A^{\prime \prime}=A \cap A x^{-1}, B^{\prime \prime}=B \cup x B\right) .
$$

The following properties are straightforward to check.

1. $A^{\prime} B^{\prime} \subset A B$ and $A^{\prime \prime} B^{\prime \prime} \subset A B$;

2. $B^{\prime} \neq \emptyset \Longleftrightarrow x \in B B^{-1}$ and $A^{\prime \prime} \neq \emptyset \Longleftrightarrow x \in A^{-1} A$;

3. $\left|A^{\prime}\right|>|A| \Longleftrightarrow A x \neq A$ and $\left|B^{\prime \prime}\right|>|B| \Longleftrightarrow x B \neq B$.

Proposition 2.7 (Kemperman [2]) Let $(A, B)$ be a pair of nonempty finite subsets in a group $G$. Assume that there is an element $d \in A^{-1} A \cap B B^{-1}$ such that either $A d \neq A$ or $d B \neq B$. Then there is a pair of nonempty subsets $\left(A_{1}, B_{1}\right)$, obtained as a Kemperman transform of $(A, B)$, satisfying:

(1) $A_{1} B_{1} \subset A B$,

(2) either $\left|A_{1}\right|+\left|B_{1}\right|=|A|+|B|$ and $\left|A_{1}\right|>|A|$, or $\left|A_{1}\right|+\left|B_{1}\right|>|A|+|B|$.

\section{A variant of a theorem of Olson}

In this section we give a variant of Theorem 2.5 which is neither stronger nor weaker, in the sense that neither result implies the other one. A linearized version of this variant will be presented in Section 8 .

Theorem 3.1 Let $A, B, C$ be nonempty finite subsets of a group $G$. Suppose $B \subset C$ and $1 \in C$. Then

$$
A B C=A B \text { or }|A B C| \geq|A|+|B| .
$$


Proof. The proof is close to that of Theorem 2.5] in [5]. If $C=\{1\}$ then $A B C=A B$. We now assume $|C| \geq 2$ and proceed by induction on $|A B|$.

If $|A B|=1$ then $|A|=|B|=1$, and $|A B C|=|C| \geq 2 \geq|A|+|B|$.

Assume now $|A B|>1$ and our statement true for any pair $\left(A_{1}, B_{1}\right)$ such that $B_{1} \subset C$ and $\left|A_{1} B_{1}\right|<|A B|$. As in Olson's original proof of Theorem 2.5. we consider two cases.

(i) Assume there exists a nonempty subset $S \subset A B$ such that $S C=S$. Since $S$ is finite, we have $S c=S$ for all $c \in C$, whence $S C^{-1}=S$.

Set $A_{0}=A \cap S$ and $A_{1}=A \backslash S$, so that $A=A_{0} \sqcup A_{1}$. We claim that

$$
A_{0} B=S .
$$

Indeed, as $B \subset C$, we have $A_{0} B \subset S C=S$. Conversely, let $s \in S$. Then $s=a b$ for some $a \in A$ and $b \in B$. Since $a=s b^{-1} \in S C^{-1}=S$, it follows that $a \in A_{0}$, whence $s \in A_{0} B$ as desired.

As a first consequence, we obtain

$$
A B=S \sqcup A_{1} B .
$$

Indeed, we have $A B=A_{0} B \cup A_{1} B=S \cup A_{1} B$. Moreover, as $S \cap A_{1}=\emptyset$, we have $S B^{-1} \cap A_{1}=\emptyset$ since $S B^{-1} \subset S C^{-1}=S$. It follows that $S \cap A_{1} B=\emptyset$.

Our next claim is that

$$
A B C=S \sqcup A_{1} B C .
$$

Indeed, we have $A B C=S C \cup A_{1} B C=S \cup A_{1} B C$. The intersection $S \cap A_{1} B C$ is indeed empty, since $S=S C^{-1}$ and so $S C^{-1} \cap A_{1} B=S \cap A_{1} B=\emptyset$.

Consequently, we derive

$$
|A B C| \geq|S|+\left|A_{1} B C\right| \geq\left|A_{0}\right|+\left|A_{1} B C\right|,
$$

where the estimate $|S| \geq\left|A_{0}\right|$ follows from the equality $S=A_{0} B$ in (1).

We may assume $A_{1} \neq \emptyset$, for otherwise $S=A_{0} B=A B$, whence $A B C=$ $S C=S=A B$ and we are done. We have $\left|A_{1} B\right|<|A B|$ by (21). By our induction hypothesis, we either have $A_{1} B C=A_{1} B$ or $\left|A_{1} B C\right| \geq\left|A_{1}\right|+|B|$.

If $A_{1} B C=A_{1} B$, then by (3) and (2), we obtain $A B C=S \cup A_{1} B C=$ $S \cup A_{1} B=A B$ and we are done in this case.

If now $\left|A_{1} B C\right| \geq\left|A_{1}\right|+|B|$, then by (4) and $|A|=\left|A_{0}\right|+\left|A_{1}\right|$, we get

$$
|A B C| \geq\left|A_{0}\right|+\left|A_{1} B C\right| \geq\left|A_{0}\right|+\left|A_{1}\right|+|B|=|A|+|B|,
$$


and we are done in this case as well.

(ii) Assume now $X C \neq X$ for every nonempty subset $X \subset A B$.

By Theorem 2.3, there exists a nonempty subset $S \subset A B$ and a subgroup $H$ such that

$$
|S| \geq|A|+|B|-|H|
$$

and $H S=S$ or $S H=S$. Let $c_{0} \in C$ be such that $S c_{0} \neq S$. We claim that

$$
\left|S \cup S c_{0}\right| \geq|S|+|H| \text {. }
$$

Indeed, if $H S=S$, then $H\left(S \cup S c_{0}\right)=S \cup S c_{0}$, and hence $S \cup S c_{0}$ is a disjoint union of left cosets of $H$. This implies (6) in this case.

On the other hand, if $S H=S$, pick $s_{0} \in S$ such that $s_{0} c_{0} \notin S$, and set $H^{\prime}=H \cup\left\{c_{0}\right\}$. In the set $S H^{\prime}$, the element $s_{0} c_{0}$ appears exactly once, since $S H \subset S$. Applying Theorem 2.2, it follows that

$$
\left|S H^{\prime}\right| \geq|S|+\left|H^{\prime}\right|-1=|S|+|H| \text {. }
$$

This implies (6) again, since $S H^{\prime}=S \cup S c_{0}$.

Now, as $\left\{1, c_{0}\right\} \subset C$, we have $S \cup S c_{0} \subset S C$, and therefore

$$
|A B C| \geq|S C| \geq\left|S \cup S c_{0}\right| \text {. }
$$

Combined with (55) and (6), this gives

$$
|A B C| \geq|S|+|H| \geq|A|+|B| \text {. }
$$

In the abelian case, the previous theorem is much shorter to prove, using Kneser's theorem, and remains true without the assumption $B \subset C$.

Theorem 3.2 Let $A, B, C$ be nonempty finite subsets of an abelian group $G$. Suppose $1 \in C$. Then

$$
A B C=A B \text { or }|A B C| \geq|A|+|B| .
$$

Proof. By Kneser's theorem, we obtain

$$
|A B| \geq|A|+|B|-|H|,
$$

where $H$ is the stabilizer of $A B$ in $G$. We have $H A B=A B$ and hence $H A B C=A B C$. In particular, both $A B$ and $A B C$ are disjoint unions of cosets of $H$. We have $A B \subset A B C$ since $1 \in C$. Therefore, if $A B C \neq A B$, it follows from the above that

$$
|A B C| \geq|A B|+|H| \geq|A|+|B| .
$$




\section{The linear setting}

From now on, and for the remainder of this paper, $K$ is a commutative field and $L$ a (possibly skew) field extension containing $K$ in its center. Given any subset $S \subset L$, we write $\langle S\rangle$ for the $K$-subspace of $L$ generated by $S$. For subsets $S_{1}, S_{2}$ of $L$, we consider the product set

$$
S_{1} S_{2}=\left\{s_{1} s_{2} \mid s_{1} \in S_{1}, s_{2} \in S_{2}\right\}
$$

and the $K$-subspace $\left\langle S_{1} S_{2}\right\rangle$ of $L$ spanned by $S_{1} S_{2}$. Note, for later use, the equality

$$
\left\langle\left\langle S_{1}\right\rangle\left\langle S_{2}\right\rangle\right\rangle=\left\langle S_{1} S_{2}\right\rangle
$$

If $S_{1}=\{x\}$, we simply write $x S_{2}$ instead of $\{x\} S_{2}$. When $S_{1}=A, S_{2}=B$ are finite-dimensional $K$-subspaces of $L$, it is easy to verify that $\langle A B\rangle$ is finite-dimensional, with

$$
\operatorname{dim}_{K}\langle A B\rangle \leq\left(\operatorname{dim}_{K} A\right)\left(\operatorname{dim}_{K} B\right)
$$

Also, for any nonzero $x \in L$, the sets $x A$ and $A x$ are $K$-subspaces of $L$, with $\operatorname{dim}_{K}(x A)=\operatorname{dim}_{K}(A x)=\operatorname{dim}_{K} A$.

Notation. For any subset $X \subset L$, we denote $X_{*}=X \backslash\{0\}$ and

$$
X_{*}^{-1}=\left\{x^{-1} \mid x \in X_{*}\right\},
$$

the set of inverses of the nonzero elements of $X$.

Note that $X_{*}^{-1} \cup\{0\}$ is not a $K$-subspace of $L$ in general, even if $X$ is.

In subsequent sections we establish linear versions of the addition theorems recalled above. In particular, we obtain lower bounds on $\operatorname{dim}_{K}\langle A B\rangle$ in terms of $\operatorname{dim}_{K} A, \operatorname{dim}_{K} B$. As for the groups setting, our main tool will be a linear version of the Kemperman transform.

\section{A linear Kemperman transform}

Let $(A, B)$ be a pair of finite-dimensional $K$-subspaces of $L$. Let $x \in L \backslash\{0\}$. We define the transformed pairs $\left(A^{\prime}, B^{\prime}\right)$ and $\left(A^{\prime \prime}, B^{\prime \prime}\right)$ with respect to $x$ as follows:

$$
\left(A^{\prime}=A+A x, B^{\prime}=B \cap x^{-1} B\right)
$$


and

$$
\left(A^{\prime \prime}=A \cap A x^{-1}, B^{\prime \prime}=B+x B\right),
$$

where + denotes the usual sum of vector subspaces. Since $A+A x=\langle A \cup A x\rangle$, we may view them as linear analogues of the classical Kemperman transforms. (Compare with Section 2.3.) They satisfy the analogous properties below:

1. $A^{\prime}, B^{\prime}, A^{\prime \prime}, B^{\prime \prime}$ are $K$-vector subspaces of $L$,

2. $\left\langle A^{\prime} B^{\prime}\right\rangle \subset\langle A B\rangle$ and $\left\langle A^{\prime \prime} B^{\prime \prime}\right\rangle \subset\langle A B\rangle$,

3. $B^{\prime} \neq\{0\} \Longleftrightarrow x \in B B_{*}^{-1}$ and $A^{\prime \prime} \neq\{0\} \Longleftrightarrow x \in A_{*}^{-1} A$,

4. $\operatorname{dim}_{K} A^{\prime}>\operatorname{dim}_{K} A \Longleftrightarrow A x \neq A$ and $\operatorname{dim}_{K} B^{\prime \prime}>\operatorname{dim}_{K} B \Longleftrightarrow x B \neq B$.

Proposition 5.1 With the same notation as above, set $D=A_{*}^{-1} A \cap B B_{*}^{-1}$. Suppose that either $A D \not \subset A$ or $D B \not \subset B$. Then there exists a pair $\left(A_{1}, B_{1}\right)$ of $K$-subspaces of $L$ satisfying:

(1) $A_{1} \neq\{0\}$ and $B_{1} \neq\{0\}$

(2) $\left\langle A_{1} B_{1}\right\rangle \subset\langle A B\rangle$,

(3) either $\operatorname{dim}_{K} A_{1}+\operatorname{dim}_{K} B_{1}=\operatorname{dim}_{K} A+\operatorname{dim}_{K} B$ and $\operatorname{dim}_{K} A_{1}>\operatorname{dim}_{K} A$, or $\operatorname{dim}_{K} A_{1}+\operatorname{dim}_{K} B_{1}>\operatorname{dim}_{K} A+\operatorname{dim}_{K} B$.

Point (3) above looks somewhat technical, but it has a very interesting and useful meaning. It says that, in $\mathbb{N}^{2}$ ordered lexicographically, one has

$$
\left(\operatorname{dim}_{K} A_{1}+\operatorname{dim}_{K} B_{1}, \operatorname{dim}_{K} A_{1}\right)>\left(\operatorname{dim}_{K} A+\operatorname{dim}_{K} B, \operatorname{dim}_{K} A\right) .
$$

This is used, for instance, in the proof of Corollary 5.2 below. Here again, note that the set $D=A_{*}^{-1} A \cap B B_{*}^{-1}$ needs not be a $K$-subspace of $L$.

Proof. Since $A D \not \subset A$ or $D B \not \subset B$, there is an element $d \neq 0$ in $D$ such that $A d \not \subset A$ or $d B \not \subset B$. Denote $p, q$ the dimensions of the following quotient spaces:

$$
p=\operatorname{dim}_{K}(A+A d) / A, \quad q=\operatorname{dim}_{K}(B+d B) / B .
$$

We have $\max (p, q) \geq 1$, and we need to distinguish two cases. 
- Assume first $p \geq q$. In this case, we make the linear Kemperman transform

$$
A_{1}=A+A d, \quad B_{1}=B \cap d^{-1} B .
$$

We have $A \subsetneq A_{1}$ since $p \geq 1$, and $B_{1} \neq\{0\}$ since $d \in D \subset B B_{*}^{-1}$. Moreover $\left\langle A_{1} B_{1}\right\rangle \subset\langle A B\rangle$. This settles the first two requirements on $\left(A_{1}, B_{1}\right)$. As for the third one, we claim that

$$
\operatorname{dim}_{K} A_{1}=\operatorname{dim}_{K} A+p, \operatorname{dim}_{K} B_{1}=\operatorname{dim}_{K} B-q .
$$

Indeed, our definitions imply $p=\operatorname{dim}_{K} A_{1}-\operatorname{dim}_{K} A$, and we have

$$
\begin{aligned}
\operatorname{dim}_{K} B_{1} & =\operatorname{dim}_{K}\left(B \cap d^{-1} B\right) \\
& =\operatorname{dim}_{K}(d B \cap B) \\
& =\operatorname{dim}_{K} d B+\operatorname{dim}_{K} B-\operatorname{dim}_{K}(d B+B) \\
& =\operatorname{dim}_{K} B-q .
\end{aligned}
$$

It follows that

$$
\operatorname{dim}_{K} A_{1}+\operatorname{dim}_{K} B_{1}=\operatorname{dim}_{K} A+\operatorname{dim}_{K} B+p-q,
$$

whence $\operatorname{dim}_{K} A_{1}+\operatorname{dim}_{K} B_{1} \geq \operatorname{dim}_{K} A+\operatorname{dim}_{K} B$, and $\operatorname{dim}_{K} A_{1}>\operatorname{dim}_{K} A$ since $p \geq 1$. This yields the third requirement on $\left(A_{1}, B_{1}\right)$.

- Assume now $p<q$. Here we use the other linear Kemperman transform and set

$$
A_{1}=A \cap A d^{-1}, \quad B_{1}=B+d B .
$$

This time we have $B \subsetneq B_{1}$ since $q \geq 1$, and $A_{1} \neq\{0\}$ since $d \in D \subset A_{*}^{-1} A$. We have $\left\langle A_{1} B_{1}\right\rangle \subset\langle A B\rangle$, and a similar calculation as above yields

$$
\operatorname{dim}_{K} A_{1}=\operatorname{dim}_{K} A-p, \operatorname{dim}_{K} B_{1}=\operatorname{dim}_{K} B+q .
$$

It follows that

$$
\operatorname{dim}_{K} A_{1}+\operatorname{dim}_{K} B_{1}=\operatorname{dim}_{K} A+\operatorname{dim}_{K} B+q-p,
$$

implying $\operatorname{dim}_{K} A_{1}+\operatorname{dim}_{K} B_{1}>\operatorname{dim}_{K} A+\operatorname{dim}_{K} B$, as desired.

Corollary 5.2 Let $A, B$ be nonzero finite-dimensional $K$-subspaces of $L$. Then there exist nonzero finite-dimensional $K$-subspaces $E, F$ of $L$ satisfying 
(1) $\langle E F\rangle \subset\langle A B\rangle$,

(2) $\operatorname{dim}_{K} E+\operatorname{dim}_{K} F \geq \operatorname{dim}_{K} A+\operatorname{dim}_{K} B$,

(3) $E D=E$ and $D F=F$, where $D=E_{*}^{-1} E \cap F F_{*}^{-1}$.

Proof. By successive applications of the previous proposition, we get a sequence

$$
(A, B)=\left(A_{0}, B_{0}\right),\left(A_{1}, B_{1}\right), \ldots,\left(A_{i}, B_{i}\right), \ldots
$$

of pairs of $K$-subspaces of $L$ satisfying the following properties, for $i \geq 1$ :

- $\left\langle A_{i} B_{i}\right\rangle \subset\left\langle A_{i-1} B_{i-1}\right\rangle$,

- $\left(\operatorname{dim}_{K} A_{i}+\operatorname{dim}_{K} B_{i}, \operatorname{dim}_{K} A_{i}\right)>\left(\operatorname{dim}_{K} A_{i-1}+\operatorname{dim}_{K} B_{i-1}, \operatorname{dim}_{K} A_{i-1}\right)$ in $\mathbb{N}^{2}$ ordered lexicographically.

Moreover, these spaces have bounded dimension, since

$$
\begin{aligned}
\max \left\{\operatorname{dim}_{K} A_{i}, \operatorname{dim}_{K} B_{i}\right\} & \leq \operatorname{dim}_{K}\left\langle A_{i} B_{i}\right\rangle \\
& \leq \operatorname{dim}_{K}\langle A B\rangle \\
& \leq\left(\operatorname{dim}_{K} A\right)\left(\operatorname{dim}_{K} B\right) .
\end{aligned}
$$

It follows that the above sequence must be finite. By Proposition 5.1, there is an index $n \geq 0$ such that the set $D=\left(A_{n}\right)_{*}^{-1} A_{n} \cap B_{n}\left(B_{n}\right)_{*}^{-1}$ satisfies $A_{n} D \subset A_{n}$ and $D B_{n} \subset B_{n}$. In fact $A_{n} D=A_{n}$ and $D B_{n}=B_{n}$, since $1 \in D$. Setting $E=A_{n}, F=B_{n}$, it follows from the properties of the sequence of $\left(A_{i}, B_{i}\right)$ that $\langle E F\rangle \subset\langle A B\rangle$ and $\operatorname{dim}_{K} E+\operatorname{dim}_{K} F \geq \operatorname{dim}_{K} A+\operatorname{dim}_{K} B$.

\section{Linearizing Theorem 2.3 of Olson}

With the linearized Kemperman transform at hand, we are now ready to establish linear versions of the addition theorems of Section 2 and 3 . We start with Theorem 2.3 of Olson. The following easy lemma will be needed in the process.

Lemma 6.1 Let $D$ be a finite-dimensional $K$-subspace of $L$. Then $D$ is a (possibly skew) field if and only if $1 \in D$ and $D^{2} \subset D$. 
Proof. It suffices to show that every nonzero element $d \in D$ is invertible in $D$. Now the map $L_{d}: D \rightarrow D$ defined by $L_{d}(x)=d x$ for all $x \in D$, is linear and injective. Hence $L_{d}$ is bijective, and therefore there is some $d^{\prime} \in D$ with $L\left(d^{\prime}\right)=1$. Obviously $d^{\prime}$ is the inverse of $d$ in $L$ and it does live in $D$.

Theorem 6.2 Let $K$ be a commutative field and $L$ a field extension of $K$. Let $A, B$ be finite-dimensional $K$-vector spaces in $L$ distinct from $\{0\}$. Then there exist a $K$-vector subspace $S$ of $\langle A B\rangle$ and a subfield $H$ of $L$ such that

(1) $K \subset H \subset L$,

(2) $\operatorname{dim}_{K} S \geq \operatorname{dim}_{K} A+\operatorname{dim}_{K} B-\operatorname{dim}_{K} H$,

(3) $H S=S$ or $S H=S$.

Proof. By Corollary 5.2, there are subspaces $E, F$ such that

$$
\langle E F\rangle \subset\langle A B\rangle, \operatorname{dim}_{K} E+\operatorname{dim}_{K} F \geq \operatorname{dim}_{K} A+\operatorname{dim}_{K} B,
$$

and $E D=E, D F=F$ where $D=E_{*}^{-1} E \cap F F_{*}^{-1}$.

We start by assuming $\operatorname{dim}_{K} E \geq \operatorname{dim}_{K} F$ and, as in the proof of Olson's theorem, we distinguish two cases:

(i) $F F_{*}^{-1} \not \subset E_{*}^{-1} E$. Then there exist $x_{1}, x_{2} \in F_{*}$ such that $x_{1} x_{2}^{-1} \notin E_{*}^{-1} E$. Therefore $E x_{1} \cap E x_{2}=\{0\}$ and $E x_{1} \oplus E x_{2} \subset\langle E F\rangle$. This gives $\operatorname{dim}_{K}\langle E F\rangle \geq$ $2 \operatorname{dim}_{K} E \geq \operatorname{dim}_{K} E+\operatorname{dim}_{K} F$. Let $H=K$ and $S=\langle E F\rangle$. Then $H$ is a subfield of $L$ stabilizing $S$, and $\operatorname{dim}_{K}\langle S\rangle \geq \operatorname{dim}_{K} A+\operatorname{dim}_{K} B-1$, as desired. (ii) $F F_{*}^{-1} \subset E_{*}^{-1} E$. Then $D=F F_{*}^{-1}$ and $1 \in D$. Moreover, we have

$$
D D=D F F_{*}^{-1}=F F_{*}^{-1}=D
$$

since $D F=F$. So $D^{2} \subset D$. Let $z$ be a nonzero element of $F$. We have

$$
F=F z^{-1} z \subset D z \subset D F=F
$$

because $F z^{-1} \subset D$ and $D F=F$. This implies that $D z=F$ and thus $D=F z^{-1}$ is a finite-dimensional $K$-vector space in $L$. Since $1 \in D$ and $D^{2}=D$, we derive from Lemma 6.1 that $D$ is a subfield of $L$ containing $K$. Moreover $\operatorname{dim}_{K} D=\operatorname{dim}_{K} F$. Set $S=\langle E F\rangle$ and $H=z^{-1} D z$. Since $D$ is a field, $H$ is also a field and $\operatorname{dim}_{K} H=\operatorname{dim}_{K} D=\operatorname{dim}_{K} F$. We have

$$
S H \subset\langle E F H\rangle=\left\langle E F z^{-1} D z\right\rangle=\left\langle E D z z^{-1} D z\right\rangle=\langle E D z\rangle=\langle E F\rangle=S
$$


since $D z=F$ and $D^{2}=D$. It follows that $S H=S$. We have $\operatorname{dim}_{K} S=$ $\operatorname{dim}_{K}\langle E F\rangle \geq \operatorname{dim}_{K} E$ since $F \neq\{0\}$. Finally, this gives

$$
\operatorname{dim}_{K} S \geq \operatorname{dim}_{K} E+\operatorname{dim}_{K} F-\operatorname{dim}_{K} H
$$

because $\operatorname{dim}_{K} H=\operatorname{dim}_{K} D=\operatorname{dim}_{K} F$.

This settles the case $\operatorname{dim}_{K} E \geq \operatorname{dim}_{K} F$. The case $\operatorname{dim}_{K} F \geq \operatorname{dim}_{K} E$ can be treated in a similar way.

\section{Remarks.}

(i) In contrast to the linear version of Kneser's Theorem 1.2, our linear version of Olson's Theorem does not require any separability hypothesis.

(ii) Assume that $L$ is commutative and $\operatorname{dim}_{K} L=p$, a prime number. In that case, there is no intermediate field $K \subset H \subset L$ besides $K, L$. It follows from the above theorem that either $\langle A B\rangle=L$ or $\operatorname{dim}_{K}\langle A B\rangle \geq \operatorname{dim}_{K} S \geq \operatorname{dim}_{K} A+\operatorname{dim}_{K} B-1$. This is also a consequence of Theorem 1.2, provided $L$ is further assumed to be separable over $K$.

(iii) Suppose that $K$ is a finite field of cardinality $q$. Then $A^{*}=A \backslash\{0\}$ and $B^{*}=B \backslash\{0\}$ are finite subsets of the group $L^{*}=L \backslash\{0\}$, and by Theorem 2.3, there exist a subset $\mathcal{S}^{*}$ of $A^{*} B^{*}$ and a subgroup $\mathcal{H}^{*}$ of $L^{*}$ such that

$$
\left|\mathcal{S}^{*}\right| \geq\left|A^{*}\right|+\left|B^{*}\right|-\left|\mathcal{H}^{*}\right| .
$$

Thus, we get

$$
|A|+|B| \leq\left|\mathcal{S}^{*}\right|+\left|\mathcal{H}^{*}\right|+2 .
$$

However, with Theorem 6.2, we obtain

$$
q^{\operatorname{dim}_{K} A} q^{\operatorname{dim}_{K} B} \leq q^{\operatorname{dim}_{K} S} q^{\operatorname{dim}_{K} H},
$$

and since $|K|=q$, this gives

$$
|A||B| \leq|S||H| .
$$

Thus, Theorem 2.3 gives an upper bound for $|A|+|B|$, whereas Theorem 6.2 gives an upper bound for $|A||B|$. Note that $\mathcal{S}^{*} \neq S \backslash\{0\}$ and $\mathcal{H}^{*} \neq H \backslash\{0\}$ in general. 
One first easy consequence is a linear version of Kemperman's Theorem 2.4 on torsion-free groups.

Theorem 6.3 Let $K$ be a commutative field and $L$ a (possibly skew) purely transcendental extension of $K$. Let $A, B$ be nonzero finite-dimensional $K$ subspaces of $L$. Then

$$
\operatorname{dim}_{K}\langle A B\rangle \geq \operatorname{dim}_{K} A+\operatorname{dim}_{K} B-1
$$

Proof. By Theorem 6.2, there is a subspace $S \subset\langle A B\rangle$ and an intermediary field $K \subset H \subset L$ such that

$$
\operatorname{dim}_{K}\langle A B\rangle \geq \operatorname{dim}_{K} A+\operatorname{dim}_{K} B-\operatorname{dim}_{K} H
$$

and $H S=S$ or $S H=S$. As $\operatorname{dim}_{K} S$ is finite, it follows that $\operatorname{dim}_{K} H$ must be finite as well. But $K$ is the only finite-dimensional subfield of $L$. It follows that $H=K$ and hence $\operatorname{dim}_{K} H=1$.

Remark. The above lower bound is sharp. Indeed, let $x \in L \backslash K$. Then $x$ is transcendental over $K$. Fix positive integers $r, s$. Let $A, B$ be the subspaces of $L$ generated by $\left\{1, x, \ldots, x^{r-1}\right\},\left\{1, x, \ldots, x^{s-1}\right\}$, respectively. Then $\operatorname{dim}_{K} A=r, \operatorname{dim}_{K} B=s$ and $\operatorname{dim}_{K}\langle A B\rangle=r+s-1$, since $\langle A B\rangle$ is the subspace spanned by the basis $\left\{1, x, \ldots, x^{r+s-2}\right\}$.

We now derive, from Theorem 6.2 again, a linear version of the basic Proposition 2.1.

Proposition 6.4 Let $K$ be a commutative field and $L$ a field extension containing $K$ in its center, with $\operatorname{dim}_{K} L$ finite. Let $A, B$ be nonzero subspaces of $L$ satisfying $\operatorname{dim}_{K} A+\operatorname{dim}_{K} B>\operatorname{dim}_{K} L$. Then $\langle A B\rangle=L$.

Note that the hypothesis that $L$ is a field is essential. For otherwise, a counterexample would be provided by $A=L$ and $B=$ a proper nonzero left ideal of $L$, yielding $\langle A B\rangle=B$.

Proof. By Theorem 6.2, there is a subspace $S \subset\langle A B\rangle$ and an intermediate field $K \subset H \subset L$ such that

$$
\operatorname{dim}_{K} S \geq \operatorname{dim}_{K} A+\operatorname{dim}_{K} B-\operatorname{dim}_{K} H,
$$


and either $H S=S$ or $S H=S$. We claim that $H S=L=S H$. Indeed, fix any nonzero element $x \in L$. It follows from (8) and the hypothesis $\operatorname{dim}_{K} A+\operatorname{dim}_{K} B>\operatorname{dim}_{K} L$, that

$$
\operatorname{dim}_{K} S+\operatorname{dim}_{K}(x H)>\operatorname{dim}_{K} L .
$$

Therefore $S \cap x H \neq\{0\}$. Hence, there are nonzero elements $s \in S$ and $h \in H$ such that $s=x h$. But then $x=s h^{-1}$ belongs to $S H$, since $h^{-1} \in H$. It follows that $L=H S$. The same argument, with $x H$ replaced by $H x$, yields $L=S H$. We conclude $L=S=\langle A B\rangle$, since $S \subset\langle A B\rangle$ and $S=H S$ or $S H$.

When $L$ is commutative, the above result admits a much simpler proof, which does not require Theorem 6.2,

Proposition 6.5 Let $K \subset L$ be a commutative field extension, with $\operatorname{dim}_{K} L$ finite. Let $A, B$ be nonzero subspaces of $L$ satisfying $\operatorname{dim}_{K} A+\operatorname{dim}_{K} B>$ $\operatorname{dim}_{K} L$. Then $\langle A B\rangle=L$.

Proof. We may assume $A, B \neq L$ and proceed by induction on $\operatorname{dim}_{K} B$. If $\operatorname{dim}_{K} B=1$, then $\operatorname{dim}_{K} A=\operatorname{dim}_{K} L$ and $\langle A B\rangle=\langle L B\rangle=L$. Assume now $\operatorname{dim}_{K} B \geq 2$. Since $\langle L B\rangle=L$, there must be a nonzero element $x \in L$ such that $x B \not \subset A$. Set

$$
A^{\prime}=A+x B, B^{\prime}=A x^{-1} \cap B .
$$

We have $A^{\prime} B^{\prime} \subset\langle A B\rangle$ by construction and the commutativity of $L$. Moreover, the subspace $B^{\prime}$ is nonzero, since $\operatorname{dim}_{K} A x^{-1}+\operatorname{dim}_{K} B=\operatorname{dim}_{K} A+$ $\operatorname{dim}_{K} B>\operatorname{dim}_{K} L$. Finally, $\operatorname{dim}_{K} A^{\prime}+\operatorname{dim}_{K} B^{\prime}=\operatorname{dim}_{K} A+\operatorname{dim}_{K} B$, since

$$
\begin{aligned}
\operatorname{dim}_{K} A^{\prime}=\operatorname{dim}_{K}(A+x B) & =\operatorname{dim}_{K} A+\operatorname{dim}_{K} x B-\operatorname{dim}_{K}(A \cap x B) \\
& =\operatorname{dim}_{K} A+\operatorname{dim}_{K} B-\operatorname{dim}_{K}\left(A x^{-1} \cap B\right) \\
& =\operatorname{dim}_{K} A+\operatorname{dim}_{K} B-\operatorname{dim}_{K} B^{\prime} .
\end{aligned}
$$

By the induction hypothesis, we conclude $\left\langle A^{\prime} B^{\prime}\right\rangle=L=\langle A B\rangle$.

Remark. We are grateful to Joseph Oesterlé for providing us with the following alternative proof of Proposition [6.4, which only uses duality in vector spaces.

Proof. Let $H$ be any hyperplane in $L$, and let $\varphi: L \rightarrow K$ be a linear form with kernel $H$. It suffices to show that there exist $a \in A, b \in B$ such that $\varphi(a b) \neq 0$, implying $\langle A B\rangle \not \subset H$. 
The map $\beta: L \times L \rightarrow K$ defined by $\beta(x, y)=\varphi(x y)$ for all $x, y \in L$ is a $K$-bilinear form, and it is non-degenerate: if $x \neq 0$, then $x L$ is equal to $L$ and hence $\varphi(x L) \neq\{0\}$. Therefore $\beta$ induces an isomorphism $\gamma: L \rightarrow L^{*}$, where $L^{*}$ is the dual of $L$, defined by the formula $\gamma(x)(y)=\beta(x, y)$ for all $x, y \in L$.

From the inclusion map $j: B \rightarrow L$ we deduce, by transposition, a surjection $j^{t}: L^{*} \rightarrow B^{*}$, defined as usual by $j^{t}(\psi)=\psi \circ j$ for all $\psi \in L^{*}$. The composition $j^{t} \circ \gamma: L \rightarrow B^{*}$ is then also surjective, and therefore

$$
\operatorname{dim}_{K} \operatorname{ker}\left(j^{t} \circ \gamma\right)=\operatorname{dim}_{K} L-\operatorname{dim}_{K} B<\operatorname{dim}_{K} A .
$$

Thus, there is some $a \in A$ satisfying $j^{t}(\gamma(a)) \neq 0$. Since the linear form $\gamma(a) \circ j: B \rightarrow K$ does not vanish, there must be some $b \in B$ satisfying $\gamma(a)(b) \neq 0$, i.e. $\beta(a, b)=\varphi(a b) \neq 0$.

\section{Linearizing Theorem 2.2 of Kemperman}

We shall now establish a linear analogue of Kemperman's Theorem 2.2, according to which, for subsets $A, B$ in a group $G$, one has $|A B| \geq|A|+|B|-1$ provided there is an element $c \in A B$ with a unique representation of the form $c=a b$ with $a \in A, b \in B$.

In order to properly linearize this result, we need to rephrase the above unicity condition on $c$. First, up to translation of $A, B$, we may assume that $1 \in A \cap B$ and that $c=1$ admits the unique representation $1=a b$ with $a=b=1$ as a product in $A B$. If we write

$$
\begin{aligned}
& A=\{1\} \sqcup \bar{A} \\
& B=\{1\} \sqcup \bar{B}
\end{aligned}
$$

with $\bar{A}, \bar{B}$ the respective complements of $\{1\}$ in $A, B$, then the unicity of 1 as a product in $A B$ is equivalent to the disjointness condition

$$
\{1\} \cap(\bar{A} \cup \bar{B} \cup \bar{A} \bar{B})=\emptyset
$$

which may also be written as $A B=\{1\} \sqcup(\bar{A} \cup \bar{B} \cup \bar{A} \bar{B})$. These equivalent conditions motivate the following formulation of our sought-for linearization. 
Theorem 7.1 Let $K$ be a commutative field and $L$ a field extension of $K$. Let $A, B$ be finite-dimensional $K$-vector spaces in $L$ such that $K \subset A \cap B$. Suppose there exist subspaces $\bar{A}, \bar{B} \subset L$ such that

$$
A=K \oplus \bar{A}, B=K \oplus \bar{B} \text { and } K \cap(\bar{A}+\bar{B}+\langle\bar{A} \bar{B}\rangle)=\{0\} .
$$

Then

$$
\operatorname{dim}_{K}\langle A B\rangle \geq \operatorname{dim}_{K} A+\operatorname{dim}_{K} B-1
$$

Proof. Observe first that, if $A=K+\bar{A}$ and $B=K+\bar{B}$, then $\langle A B\rangle=$ $K+(\bar{A}+\bar{B}+\langle\bar{A} \bar{B}\rangle)$. Therefore, condition (9) is equivalent to

$$
A=K \oplus \bar{A}, B=K \oplus \bar{B} \text { and }\langle A B\rangle=K \oplus(\bar{A}+\bar{B}+\langle\bar{A} \bar{B}\rangle) .
$$

Since $1 \in A \cap B$, we have $A+B \subset\langle A B\rangle$. We now distinguish two cases.

Case 1. Assume $A \cap B=K$. Then $\operatorname{dim}_{K}(A+B)=\operatorname{dim}_{K} A+\operatorname{dim}_{K} B-1$, and we are done since $\operatorname{dim}_{K}\langle A B\rangle \geq \operatorname{dim}_{K}(A+B)$.

Case 2. Assume now $A \cap B \neq K$, i.e. $\operatorname{dim}_{K} A \cap B \geq 2$. We first claim that

$$
A \cap B=K \oplus(\bar{A} \cap \bar{B}) .
$$

Indeed, let $x \in A \cap B$. Then there are $\lambda, \mu \in K$ and $\bar{x} \in \bar{A}, \bar{y} \in \bar{B}$ such that

$$
x=\lambda+\bar{x}=\mu+\bar{y} .
$$

Since $K \cap(\bar{A}+\bar{B})=\{0\}$, it follows that $\lambda=\mu$ and $\bar{x}=\bar{y}$, so that $x \in$ $K \oplus(\bar{A} \cap \bar{B})$, as claimed. The reverse inclusion is immediate.

Observe that in the present case, we have $\bar{A} \cap \bar{B} \neq\{0\}$. We shall perform a suitable sequence of linear Kemperman transforms on the pair $(A, B)$ and eventually reach Case 1 again, thereby concluding the proof.

Let $0 \neq d \in \bar{A} \cap \bar{B}$ be any nonzero element. We perform a Kemperman transform on $(A, B)$ relative to $d$, and get a new pair $\left(A_{1}, B_{1}\right)$, by defining either

$$
\begin{aligned}
& \text { (i) } \quad A_{1}=A+A d, \quad B_{1}=B \cap d^{-1} B \text {, or } \\
& \text { (ii) } A_{1}=A \cap A d^{-1}, \quad B_{1}=B+d B \text {. }
\end{aligned}
$$

In either case, we have $\left\langle A_{1} B_{1}\right\rangle \subset\langle A B\rangle$, and one of $(i)$ or $(i i)$ will yield the estimate

$$
\operatorname{dim}_{K} A_{1}+\operatorname{dim}_{K} B_{1} \geq \operatorname{dim}_{K} A+\operatorname{dim}_{K} B
$$


Case $(i)$. For any nonzero element $d \in \bar{A} \cap \bar{B}$, define

$$
\begin{aligned}
& A_{1}=A+A d, \\
& B_{1}=B \cap d^{-1} B .
\end{aligned}
$$

We shall show that this new pair satisfies the hypotheses of the theorem. Indeed, define

$$
\begin{aligned}
& \overline{A_{1}}=\bar{A}+A d, \\
& \overline{B_{1}}=\bar{B} \cap d^{-1} \bar{B} .
\end{aligned}
$$

We claim that conditions (9) are satisfied, i.e.

$$
\begin{aligned}
A_{1} & =K \oplus \overline{A_{1}}, \\
B_{1} & =K \oplus \overline{B_{1}}, \\
\{0\} & =K \cap\left(\overline{A_{1}}+\overline{B_{1}}+\left\langle\overline{A_{1}} \overline{B_{1}}\right\rangle\right) .
\end{aligned}
$$

For (13): We have $K+\overline{A_{1}}=K+\bar{A}+A d=A+A d=A_{1}$. The sum is direct, since $\overline{A_{1}} \subset \bar{A}+K d+\bar{A} d \subset \bar{A}+\bar{B}+\langle\bar{A} \bar{B}\rangle$, whence $K \cap \overline{A_{1}}=\{0\}$ by (9).

For (14): To start with, we have $K \cap \overline{B_{1}} \subset K \cap \bar{B}=\{0\}$ by (9).

We next verify the inclusion $K+\overline{B_{1}} \subset B_{1}$. We have $K \subset B$, and $K \subset$ $d^{-1} B$ since $d \in B$, whence $K \subset B_{1}$. Moreover, we have $\overline{B_{1}}=\bar{B} \cap d^{-1} \bar{B} \subset$ $B \cap d^{-1} B=B_{1}$. This establishes the desired inclusion.

It remains to prove the reverse inclusion $B_{1} \subset K+\overline{B_{1}}$. Let $x \in B_{1}=$ $B \cap d^{-1} B$. Since $B=K \oplus \bar{B}$, we may write $x=\lambda+\bar{x}$ for some $\lambda \in K$ and $\bar{x} \in \bar{B}$. It remains to show that $\bar{x} \in \overline{B_{1}}=\bar{B} \cap d^{-1} \bar{B}$, i.e. that $\bar{x} \in d^{-1} \bar{B}$. Since $x \in d^{-1} B$, there are elements $y \in B, \mu \in K$ and $\bar{y} \in \bar{B}$ such that $x=d^{-1} y$ and $y=\mu+\bar{y}$. Hence $d x=y$, and therefore

$$
d \lambda+d \bar{x}=\mu+\bar{y}
$$

It follows that $\mu=d \lambda+d \bar{x}-\bar{y}$, whence $\mu \in K \cap(\bar{B}+\langle\bar{A} \bar{B}\rangle)$. Therefore $\mu=0$ by (9), whence $d \bar{x}=\bar{y}-d \lambda \in \bar{B}$. This shows that $\bar{x} \in d^{-1} \bar{B}$, implying $\bar{x} \in \overline{B_{1}}$ and finally $x \in K+\overline{B_{1}}$, as desired.

For (15): By (9), it suffices to show the inclusion

$$
\left(\overline{A_{1}}+\overline{B_{1}}+\left\langle\overline{A_{1}} \overline{B_{1}}\right\rangle\right) \subset(\bar{A}+\bar{B}+\langle\bar{A} \bar{B}\rangle) .
$$


Considering each summand at a time in the left-hand side, we have:

- $\overline{A_{1}}=\bar{A}+A d=\bar{A}+K d+\bar{A} d \subset(\bar{A}+\bar{B}+\langle\bar{A} \bar{B}\rangle)$,

- $\overline{B_{1}}=\bar{B} \cap d^{-1} \bar{B} \subset \bar{B}$,

- $\overline{A_{1}} \overline{B_{1}}=(\bar{A}+A d)\left(\bar{B} \cap d^{-1} \bar{B}\right) \subset \bar{A} \bar{B}+A \bar{B} \subset \bar{A} \bar{B}+\bar{B}+\bar{A} \bar{B}$,

and we are done.

Case $(i i)$. For any nonzero element $d \in \bar{A} \cap \bar{B}$, define

$$
\begin{aligned}
& A_{1}=A \cap A d^{-1}, \\
& B_{1}=B+d B .
\end{aligned}
$$

This time, we set

$$
\begin{aligned}
& \overline{A_{1}}=\bar{A} \cap \bar{A} d^{-1}, \\
& \overline{B_{1}}=\bar{B}+d B .
\end{aligned}
$$

With arguments similar to those of Case $(i)$, we can prove that conditions (9) are satisfied again, i.e.

$$
\begin{aligned}
A_{1} & =K \oplus \overline{A_{1}}, \\
B_{1} & =K \oplus \overline{B_{1}}, \\
\{0\} & =K \cap\left(\overline{A_{1}}+\overline{B_{1}}+\left\langle\overline{A_{1}} \overline{B_{1}}\right\rangle\right) .
\end{aligned}
$$

Now, as in the proof of Corollary [5.2, we iterate the above Kemperman transforms as long as possible. At each step, we get new subspaces $A_{i}, B_{i}$ satisfying (9) and such that

$$
\left(\operatorname{dim}_{K} A_{i}+\operatorname{dim}_{K} B_{i}, \operatorname{dim}_{K} A_{i}\right)>\left(\operatorname{dim}_{K} A_{i-1}+\operatorname{dim}_{K} B_{i-1}, \operatorname{dim}_{K} A_{i-1}\right)
$$

in $\mathbb{N}^{2}$ ordered lexicographically. Since these subspaces have bounded dimension, the iteration cannot continue indefinitely and Case 1 must eventually be reached. This means that there exist subspaces $E, F$ of $L$ such that

$$
\begin{aligned}
\langle E F\rangle & \subset\langle A B\rangle, \\
\operatorname{dim}_{K} E+\operatorname{dim}_{K} F & \geq \operatorname{dim}_{K} A+\operatorname{dim}_{K} B,
\end{aligned}
$$

and satisfying $E \cap F=K$ together with the hypotheses of the theorem. By Case 1, we have

$$
\operatorname{dim}_{K}\langle E F\rangle \geq \operatorname{dim}_{K} E+\operatorname{dim}_{K} F-1 .
$$

The desired inequality, namely

$$
\operatorname{dim}_{K}\langle A B\rangle \geq \operatorname{dim}_{K} A+\operatorname{dim}_{K} B-1,
$$

now follows from (16) and (17). 


\section{Linearizing Theorem 3.1}

Throughout the last two sections, we shall assume that $L$ is a commutative field extension of $K$ and that every algebraic element of $L$ is separable over $K$. This allows us to use Theorem 1.2, the linear version of Kneser's Theorem.

Our results below probably remain true in the more general setting of the preceding sections, where $L$ is only assumed to be a field containing $K$ in its center. But we have no proof of this so far.

Lemma 8.1 Let $K$ be a commutative field, $H$ a field extension of $K$ and $L$ a field extension of $H$. Let $V$ be a finite-dimensional $K$-vector space in $L$ such that $H V=V$. Then there exists a finite subset $R_{V} \subset V$ such that

$$
V=\bigoplus_{v \in R_{V}} H v
$$

In particular $\operatorname{dim}_{K} H$ is finite and divides $\operatorname{dim}_{K} V$.

Proof. For any $v \in V, H v$ is vector subspace of $V$. Moreover for any $v, v^{\prime}$ in $V$, one has $H v=H v^{\prime}$ or $H v \cap H v^{\prime}=\{0\}$. The lemma follows immediately since $\operatorname{dim}_{K} V$ is finite.

Remark. If $H V=V$, then $V$ can be interpreted as a finite-dimensional left $H$-module, and (18) gives its decomposition into irreducible components.

We now give a linear version of Theorem 3.1. As mentioned above, the hypotheses on $L$ are probably more restrictive than actually necessary.

Theorem 8.2 Let $K \subset L$ be commutative fields such that every algebraic element in $L$ is separable over $K$. Let $A, B, C \subset L$ be finite-dimensional $K$-subspaces of $L$ such that $A, B \neq\{0\}$ and $K \subset C$. Then either

$$
\langle A B C\rangle=\langle A B\rangle \text { or } \operatorname{dim}_{K}\langle A B C\rangle \geq \operatorname{dim}_{K} A+\operatorname{dim}_{K} B .
$$

Proof. We shall apply Theorem 1.2. The stabilizer $H$ of $\langle A B\rangle$ is a field extension of $K$, and we have

$$
\operatorname{dim}_{K}\langle A B\rangle \geq \operatorname{dim}_{K} A+\operatorname{dim}_{K} B-\operatorname{dim}_{K} H .
$$

Then $H$ stabilizes $\langle A B C\rangle$, since $H\langle A B\rangle c=\langle A B\rangle c$ for all $c \in C$. Of course $\langle A B\rangle$ is a subspace of $\langle A B C\rangle$, since $1 \in C$. Assume $\langle A B C\rangle \neq\langle A B\rangle$. Since 
$H\langle A B C\rangle=\langle A B C\rangle$, Lemma 8.1 implies the existence of a nonempty finite subset $R \subset\langle A B C\rangle$ such that $R \cap\langle A B\rangle=\emptyset$ and

$$
\langle A B C\rangle=\langle A B\rangle \oplus \bigoplus_{v \in R} H v .
$$

In particular, we have

$$
\operatorname{dim}_{K}\langle A B C\rangle=\operatorname{dim}_{K}\langle A B\rangle+|R| \operatorname{dim}_{K} H .
$$

Since $|R|>0$, this gives

$$
\operatorname{dim}_{K}\langle A B C\rangle \geq \operatorname{dim}_{K}\langle A B\rangle+\operatorname{dim}_{K} H .
$$

Finally, by (19), we obtain the desired inequality

$$
\operatorname{dim}_{K}\langle A B C\rangle \geq \operatorname{dim}_{K} A+\operatorname{dim}_{K} B .
$$

Corollary 8.3 Let $K \subset L$ be commutative fields such that every algebraic element in $L$ is separable over $K$. Let $A, B$ be nonzero finite-dimensional $K$-subspaces of $L$. Then either

$$
\left\langle A B B_{*}^{-1} B\right\rangle=\langle A B\rangle \text { or } \operatorname{dim}_{K}\left\langle A B^{2}\right\rangle \geq \operatorname{dim}_{K} A+\operatorname{dim}_{K} B .
$$

Proof. Assume $\left\langle A B B_{*}^{-1} B\right\rangle \neq\langle A B\rangle$. Then there exists $b_{0} \in B$ such that $\left\langle A B b_{0}^{-1} B\right\rangle \neq\langle A B\rangle$. Thus $\left\langle A B b_{0}^{-1} B b_{0}^{-1}\right\rangle \neq\left\langle A B b_{0}^{-1}\right\rangle$. We have $1 \in B b_{0}^{-1}$. Thus, applying the above theorem to $A$ and $B b_{0}^{-1}$, we get

$$
\operatorname{dim}_{K}\left\langle A B^{2}\right\rangle=\operatorname{dim}_{K}\left\langle A B b_{0}^{-1} B b_{0}^{-1}\right\rangle \geq \operatorname{dim}_{K} A+\operatorname{dim}_{K} B .
$$

\section{Powers of subspaces}

As in the preceding section, we assume that $L$ is a commutative field extension of $K$ in which every algebraic element is separable over $K$. If $B$ is a nonzero finite-dimensional $K$-subspace of $L$, we shall consider the sequence of powers $B,\left\langle B^{2}\right\rangle,\left\langle B^{3}\right\rangle, \ldots$ and analyze the evolution of the nondecreasing 
sequence $\operatorname{dim}_{K}\left\langle B^{i}\right\rangle, i \geq 1$. Without loss of generality, replacing $B$ by $b^{-1} B$ for some $b \in B \backslash\{0\}$, we may and will assume that $B$ contains 1 . Under this hypothesis, the sequence $\left\langle B^{i}\right\rangle$ turns into an ascending chain

$$
B \subset\left\langle B^{2}\right\rangle \subset\left\langle B^{3}\right\rangle \subset \ldots
$$

This chain may eventually stabilize at $\left\langle B^{n}\right\rangle$ for some $n \geq 1$, for instance if $L$ is finite-dimensional over $K$. We start by analyzing the least such exponent $n$, if any.

Proposition 9.1 Let $K$ be a commutative field and $L$ a field extension of $K$ containing $K$ in its center. Let $B$ be a finite-dimensional $K$-subspace of $L$ containing 1 . Let $n \geq 1$. The following are equivalent:

(1) $\left\langle B^{n+1}\right\rangle=\left\langle B^{n}\right\rangle$;

(2) $\left\langle B^{2 n}\right\rangle=\left\langle B^{n}\right\rangle$;

(3) $\left\langle B^{n}\right\rangle$ is a field.

Proof. First observe that, if $U, V$ are any subsets of $L$, then

$$
\langle U V\rangle=\langle\langle U\rangle\langle V\rangle\rangle
$$

Indeed, both $K$-subspaces are generated by the subset $U V$. In particular, we may and will freely use formulas such as $\left\langle B^{m}\right\rangle=\left\langle\left\langle B^{i}\right\rangle\left\langle B^{m-i}\right\rangle\right\rangle$ for integers $0 \leq i \leq m$.

Assume first $\left\langle B^{n+1}\right\rangle=\left\langle B^{n}\right\rangle$. Then we claim that $\left\langle B^{n+i}\right\rangle=\left\langle B^{n}\right\rangle$ for all $i \geq 1$. Indeed, proceeding by induction on $i$, we have

$$
\left\langle B^{n+i}\right\rangle=\left\langle\left\langle B^{n+i-1}\right\rangle B\right\rangle=\left\langle B^{n+1}\right\rangle=\left\langle B^{n}\right\rangle .
$$

For $i=n$, this gives $\left\langle B^{2 n}\right\rangle=\left\langle B^{n}\right\rangle$. In turn, this equality is equivalent to $\left\langle\left\langle B^{n}\right\rangle\left\langle B^{n}\right\rangle\right\rangle=\left\langle B^{n}\right\rangle$. By Lemma 6.1, it follows that $\left\langle B^{n}\right\rangle$ is a field.

Conversely, if $\left\langle B^{n}\right\rangle$ is a field, then $\left\langle B^{2 n}\right\rangle=\left\langle\left\langle B^{n}\right\rangle\left\langle B^{n}\right\rangle\right\rangle=\left\langle B^{n}\right\rangle$. As $\left\langle B^{n}\right\rangle \subset\left\langle B^{n+1}\right\rangle \subset\left\langle B^{2 n}\right\rangle$, this implies that $\left\langle B^{n+1}\right\rangle=\left\langle B^{n}\right\rangle$.

In particular, the smallest integer $n \geq 1$, if any, such that $\left\langle B^{n}\right\rangle=\left\langle B^{n+1}\right\rangle$ coincides with the smallest integer $n \geq 1$, if any, such that $\left\langle B^{n}\right\rangle$ is a field. 
Theorem 9.2 Let $K$ be a commutative field and $L$ a commutative field extension of $K$. Suppose that every algebraic element in $L$ is separable over $K$. Let $B$ be a finite-dimensional $K$-vector space in $L$ containing 1 , and let $n \geq 1$. Then either

$$
\left\langle B^{n+1}\right\rangle=\left\langle B^{n}\right\rangle
$$

or

$$
\operatorname{dim}_{K}\left\langle B^{n+1}\right\rangle \geq \operatorname{dim}_{K}\left\langle B^{n-1}\right\rangle+\operatorname{dim}_{K} B .
$$

Proof. Observe first that $\left\langle B^{n+1}\right\rangle=\left\langle B^{n}\right\rangle$ if and only if $\left\langle B^{n+1}\right\rangle=\left\langle B^{n} b\right\rangle$ for all $b \in B \backslash\{0\}$. Indeed, we have $\left\langle B^{n}\right\rangle \subset\left\langle B^{n} b\right\rangle \subset\left\langle B^{n+1}\right\rangle$, and $\left\langle B^{n+1}\right\rangle$ is the sum of all the subspaces $\left\langle B^{n} b\right\rangle$ where $b$ runs over $B \backslash\{0\}$.

It follows that $\left\langle B^{n+1}\right\rangle=\left\langle B^{n}\right\rangle$ is equivalent to $\left\langle B^{n} b_{1}\right\rangle=\left\langle B^{n} b_{2}\right\rangle$ for all $b_{1}, b_{2} \in B \backslash\{0\}$, which in turn is equivalent to $\left\langle B^{n}\left(B B_{*}^{-1}\right)\right\rangle=\left\langle B^{n}\right\rangle$.

Assume now $\left\langle B^{n}\left(B B_{*}^{-1}\right)\right\rangle \neq\left\langle B^{n}\right\rangle$. Since $L$ is a commutative field, this is equivalent to $\left\langle B^{n}\left(B_{*}^{-1} B\right)\right\rangle \neq\left\langle B^{n}\right\rangle$. Applying Corollary 8.3 to $B^{n-1}$ and $B$, this gives

$$
\operatorname{dim}_{K}\left\langle B^{n+1}\right\rangle \geq \operatorname{dim}_{K}\left\langle B^{n-1}\right\rangle+\operatorname{dim}_{K} B,
$$

as required.

Corollary 9.3 Let $K$ be a commutative field and $L$ a finite separable commutative extension of $K$. Let $B$ be a $K$-vector space in $L$ containing 1 . Then the smallest integer $n \geq 1$ such that $\left\langle B^{n}\right\rangle$ is a field satisfies

$$
n \leq 2 \operatorname{dim}_{K} L / \operatorname{dim}_{K} B .
$$

Proof. By Proposition 9.1, we have $B \varsubsetneqq\left\langle B^{2}\right\rangle \varsubsetneqq \cdots \varsubsetneqq\left\langle B^{n}\right\rangle$. It follows from Theorem 9.2 that

$$
\operatorname{dim}_{K}\left\langle B^{n}\right\rangle \geq\left\{\begin{aligned}
\frac{(n+1)}{2} \operatorname{dim}_{K} B & \text { if } n \text { is odd, } \\
\frac{n}{2} \operatorname{dim}_{K} B & \text { if } n \text { is even. }
\end{aligned}\right.
$$

Since $\operatorname{dim}_{K}\left\langle B^{n}\right\rangle \leq \operatorname{dim}_{K} L$, this imposes the inequality

$$
n \leq \begin{cases}2 \operatorname{dim}_{K} L / \operatorname{dim}_{K} B-1 & \text { if } n \text { is odd, } \\ 2 \operatorname{dim}_{K} L / \operatorname{dim}_{K} B & \text { if } n \text { is even. }\end{cases}
$$


Remark. From the previous Corollary, we deduce

$$
n \leq\left\lfloor\frac{2 \operatorname{dim}_{K} L}{\operatorname{dim}_{K} B}\right\rfloor .
$$

This upper bound is sharp. This can easily be seen, for example by choosing for $B$ a supplementary space of $K$ in $L$.

Acknowledgment. The authors thank Vincent Fleckinger, Joseph Oesterlé and Surya D. Ramana for stimulating discussions concerning Section [6 of this paper.

\section{References}

[1] X. D. Hou, K. H. Leung and Xiang. Q, A generalization of an addition theorem of Kneser, J. Number Theory 97 (2002), 1-9.

[2] J. H. B. Kemperman, On complexes in a semigroup, Indag. Math 18 (1956), 247-254.

[3] M. KNESER, Abschätzung der asymptotischen Dichte von Summenmengen, Math. Z. 58 (1953), 459-484.

[4] M. Kneser, Ein Satz über abelsche Gruppen mit Anwendungen auf die Geometrie der Zahlen, Math. Z. 61 (1955), 429-434.

[5] J. E. Olson, On the sum of two sets in a group, J. Number Theory $\mathbf{1 8}$ (1984), 110-120. 